\title{
Regional Differences in Chronic Stress-induced Alterations in Mast Cell and Protease-activated Receptor-2-positive Cell Numbers in the Colon of Ws/Ws Rats
}

\author{
Yong Sung Kim, ${ }_{1,2}$ Moon Young Lee, ${ }^{1,3}$ Han Seung Ryu ${ }^{1,4}$ Eul-Sig Choi, ${ }^{1,3}$ Jung Taek Oh, ${ }^{1,5}$ Ki Jung Yun ${ }^{1,6}$ and \\ Suck Chei Choi ${ }^{1,4 *}$ \\ ${ }^{1}$ Digestive Disease Research Institute, Wonkwang University School of Medicine, Iksan, Jeollabuk-do, Korea; ' 2 Department of Gastroenterology, \\ Wonkwang University Sanbon Hospital, Gunpo, Gyeonggi-do, Korea; ${ }^{3}$ Department of Physiology and Institute of Wonkwang Medical Science, \\ Wonkwang University School of Medicine, Iksan, Jeollabuk-do, Korea; and Departments of ${ }^{4}$ Gastroenterology, ${ }^{5}$ Surgery and ${ }^{6}$ Pathology, \\ Wonkwang University School of Medicine, Iksan, Jeollabuk-do, Korea
}

\section{Background/Aims}

There have been no reports on the effect of chronic psychological stress on colonic immune cells or the regional differences. We aimed to investigate the effect of chronic psychological stress on the number of mast cells and protease-activated receptor (PAR)-2-positive cells in the rat colonic mucosa.

\section{Methods}

Six-week-old and 14-week-old Ws/Ws rats, which lack mast cells after 10 weeks, were used as control and mast cell-deficient groups, respectively. The rats were divided into stress and sham-treated groups. Rats in the stressed group were exposed to water avoidance stress (WAS, 1 hour/day) for 13 days. Fecal pellet output and the number of mast cells and PAR-2-positive cells in colonic mucosa were compared between the WAS and sham groups.

\section{Results}

In 6-week-old rats, the WAS group showed a significantly higher number of mast cells compared to the sham group. In 14-week-old rats, mast cells were nearly absent in the colonic mucosa. WAS significantly increased PAR-2-positive cells in 14-week-old rats, but not in 6-week-old rats. Indirect estimation of PAR-2-positive mast cells in 6-week-old rats suggested that the majority of increased mast cells following WAS did not express PAR-2. WAS increased mast cells and PAR-2-positive cells mainly in the proximal colon. Fecal pellet output was continuously higher in the WAS group than in the sham group, and the difference was significant for both 6 -week-old and 14-week-old rats.

\section{Conclusions}

Chronic psychological stress increased the number of mast cells and PAR-2-positive cells in rat colonic mucosa, and these in-

Received: October 15, 2013 Revised: November 22, 2013 Accepted: November 23, 2013

(c) This is an Open Access article distributed under the terms of the Creative Commons Attribution Non-Commercial License (http://creativecommons. org/licenses/by-nc/3.0) which permits unrestricted non-commercial use, distribution, and reproduction in any medium, provided the original work is properly cited.

*Correspondence: Suck Chei Choi, MD, PhD

Division of Gastroenterology, Department of Internal Medicine, Wonkwang University School of Medicine, 460, Iksan-daero, Iksan, Jeollabuk-do 570-749, Korea

Tel: +82-63-859-2563, Fax: +82-63-855-2025, E-mail: medcsc@wmc.wonkwang.ac.kr

Financial support: This study was supported by Sungsan fund of Wonkwang University 2009 (YS Kim) and the Korean Society of Neurogastroenterology and Motility (SC Choi).

Conflicts of interest: None.

Author contributions: YS Kim and SC Choi designed the research; YS Kim, MY Lee, ES Choi, HS Ryu, JT OH and KJ Yun analysed and interpreted data; SC Choi, MY Lee and HS Ryu participated in drafting the article; YS Kim wrote and revised this paper; SC Choi gave final approval of the version to be published. 
creases were more prominent in the proximal colon.

\section{(J Neurogastroenterol Motil 2014;20:54-63)}

\section{Key Words}

Colon; Mast cells; Rats; Receptor, PAR-2; Stress, psychological

\section{Introduction}

Mast cells are known to play a pivotal role in allergic disease. Recently, increasing evidence has shown that mast cells are important in the pathophysiology of irritable bowel syndrome (IBS). ${ }^{1}$ Increased numbers of mast cells have been found in the gastrointestinal (GI) tract of patients with IBS. ${ }^{2,3}$ In the colon, mast cells in close proximity to nerves are significantly correlated with the severity and frequency of abdominal pain and discomfort in patients with IBS. ${ }^{4}$ However, the area in the GI tract with increased mast cells differs, and increases have been reported in the cecum, descending colon, rectum, terminal ileum and jejunum from different studies. ${ }^{2-7}$ Tryptase released from mast cells can interact with protease-activated receptor (PAR)-2. ${ }^{8}$ PAR-2 agonists induce colonic smooth muscle relaxation, activation of spinal afferent neurons, delayed rectal hyperalgesia, and changes in the intestinal permeability of rats, suggesting that PAR-2 plays some role in the pathogenesis of IBS. ${ }^{9,10}$ Stress has been known to increase immune activation in IBS, ${ }^{11}$ and acute restraint stress increases mast cell and PAR-2-positive cell numbers in the colonic mucosa of Wistar rats. ${ }^{12}$ However, there have been no reports on the effect of chronic psychological stress on these cells or the regional differences within the rat colon. Because mast cells also express PAR-2, it is preferable to use mast cell-deficient rats to investigate alterations in other PAR-2 cells besides mast cells. Rats with a homozygous mutation at the white spotting (Ws) locus (Ws/Ws rats) have a mutation in the $c$-kit $\mathrm{cDNA}^{13}$ and become deficient in mast cells after 10 weeks of age. ${ }^{14,15}$ Because the $c$-kit gene affects the development of the interstitial cells of Cajal (ICC), not only are mast cells absent from the GI tract, but ICC in the myenteric plexus are also not found in $\mathrm{Ws} / \mathrm{Ws}$ rats. ${ }^{16}$ Therefore, the ICC deficiency in Ws/Ws rats could be a confounding factor in certain experiments. To avoid this confounding effect, young Ws/Ws rats, which have normal mast cells, can be used as a control rat instead of a wild-type rat. However, there have been no comparative studies on the effects of chronic psychological stress on colonic motility and histological changes us- ing young and old Ws/Ws rats.

Therefore, we aimed to investigate whether repeated chronic psychological stress increases mast cells and PAR-2-positive cells in the colonic mucosa, and if the alterations in the proximal and distal colon differ. We used young (6-week-old) Ws/Ws rats as a control group to determine whether the colonic motility response to chronic psychological stress differs between 6-week-old and 14-week-old Ws/Ws rats.

\section{Materials and Methods}

\section{Animals}

Six-week-old $(\mathrm{n}=12)$ and 14-week-old $(\mathrm{n}=12)$ homozygous "white spotting" mutant rats (Ws/Ws rats) ${ }^{15}$ were purchased from SLC (Japan SLC, Inc., Tokyo, Japan). Ws/Ws rats have a 12-base deletion in the tyrosine kinase domain of the $c$-kit cDNA and are deficient in both mucosal mast cells and connective tissue type mast cells. ${ }^{13}$ Mast cells are nearly absent after 10 weeks of age and are completely absent after 50 weeks of age. ${ }^{14,15}$ In addition, ICC in the myenteric plexus are also not found in Ws/Ws rats because of the mutation in the $c$-kit gene, resulting in colonic contractile patterns that differ from that of wild-type rats. ${ }^{16} \mathrm{Ws} / \mathrm{Ws}$ rats aged over 10 weeks and wild-type rats are usually used in experiments investigating the effect of mast cells. In the present experiment, we used 6-week-old rats as a control group and 14-week-old rats as the mast cell-deficient group. Therefore, the PAR-2-positive cells found in 14-week-old Ws/Ws rats would not include mast cells.

Rats were housed in individual cages for 7 days to acclimate them to the temperature-controlled environment of $24^{\circ} \mathrm{C}$ with 12-hours of light/dark cycle before initiation of the stress experiment. They had free access to food (Purina Rat Chow no. 5008; Ralston Purina, St. Louis, MO, USA) and tap water during the acclimation period. Animal use protocols were approved by the Committee on Animal Research at Wonkwang University, and all efforts were made to minimize animal suffering. 


\section{Stress Protocol}

Rats were handled by the same person and subjected to water avoidance stress (WAS, 1 hour/d) for 13 days. ${ }^{11}$ WAS is a well-known psychological stress protocol in rats and can be applied as an acute single session or as chronic, repeated sessions. ${ }^{11,17}$ WAS has been reported to increase fecal pellet output (FPO), colonic mast cells, mucosal cytokines, and intestinal permeability and induce visceral hypersensitivity. ${ }^{11,17,18}$ After acclimation, rats were randomly divided into 4 groups according to age and exposure to stress: (1) 6-week-old sham stress group, (2) 14-week-old sham stress group, (3) 6-week-old stress group and (4) 14-week-old stress group. The WAS protocol was conducted by placing a single rat on a glass platform $(10 \times 8 \times 8 \mathrm{~cm})$ anchored on the center of a standard plastic cage $(45 \mathrm{~cm}$ [length] $\times$ $25 \mathrm{~cm}$ [width] $\times 25 \mathrm{~cm}$ [height] ) filled with warm water $\left(25^{\circ} \mathrm{C}\right)$ to $1 \mathrm{~cm}$ below the height of the platform for 1 hour. Rats in the sham WAS group were placed on the same platform anchored to a container without water for 1 hour.

\section{Measurement of Colonic Motility}

The body weight of each animal was measured every morning during the experimental period. We used a previously reported, validated procedure to measure the motility of the colon. ${ }^{17}$ Rats were exposed to WAS or the sham treatment for 1 hour in the morning each day and the expelled fecal pellets in the tank were counted at the end of each 1-hour session.

\section{Immunohistochemistry}

Rats were euthanized after completion of the experiment. The whole colon was removed and then divided into the proximal and distal portions. The specimens were fixed with masked formalin 2A (20\% neutral-buffered formalin), and the paraffin block containing the embedded tissues was cut into $4-\mu \mathrm{m}$ thick sections and mounted on a slide glass. Then, the tissue sections were processed with a fully automatic IHC \& ISH staining system (Ventana Medical System, Inc., Tucson, AZ, USA) for either hematoxylin histology or immunohistochemistry. The slides were incubated with the primary antibodies, a mouse monoclonal anti-mast cell tryptase (Lab Vision, Fremont, CA, USA) and a rabbit polyclonal anti-PAR-2 (Millipore, Bedford, MA, USA) antibody, for 32 minutes at $42^{\circ} \mathrm{C}$. For the latter, we used an iView DAB detection kit (Ventana Medical System, Inc.), which is composed of a biotinylated Ig secondary antibody, Streptavidin-horseradish peroxidase (SA-HRP), 3, 3'-diaminobenzidine tetrahydro- chloride (DAB) substrate, $\mathrm{H}_{2} \mathrm{O}_{2}$ and copper. Sections were then counterstained with hematoxylin (Ventana Medical System, Inc.) to stain the nucleus for 4 minutes, then dehydrated, cleared, and mounted in synthetic mountant (Shandon, Pittsburgh, PA, USA). Mast cells and PAR-2-positive cells were quantified by the same researcher without knowledge of the animal groupings. Photographs of tryptase or PAR-2-positive cells were taken of 8 contiguous non-overlapping fields on 4 immunostained slides per rat (the total number of photographs was 32 per rat) under a microscope with a digital camera system (Olympus BX50 microscope and DP70 digital camera; Tokyo, Japan). The number of cells stained with the primary antibody was counted in all photographs, and cell numbers were expressed as the mast cells or PAR-2-positive cells per high power field (number of cells/hpf).

\section{Statistical Analyses}

The results are expressed as the mean \pm SEM. We compared the FPO and the number of mast cells and PAR-2-positive cells between the WAS group and the sham group in both the 6and 14-week-old rats using repeated measured ANOVA and Student's $t$ test. Statistical analyses were performed using SPSS software version 16.0 (SPSS Inc., Chicago, IL, USA). When several observations were obtained from the same rat, such as mast cell or PAR-2-positive cell counts from contiguous, non-overlapping fields, the mean value for each rat was calculated before the average of group was obtained, i.e., $\mathrm{n}$ values represent the number of rats. A $P$-value of less than 0.05 was considered statistically significant.

\section{Results}

\section{Change in Mast Cell Numbers in the Colon}

In 6-week-old Ws/Ws rats, WAS for 13 days was associated with a significantly higher number of mast cells in the mucosa of the colon than in the sham group (11.84 \pm 0.98 vs. $7.69 \pm 0.81$, $P<0.01$; Fig. 1A, 1B and 2A). In 14-week-old Ws/Ws rats, mast cells were nearly absent in the colonic mucosa, and WAS did not affect the number of mast cells ( $0.46 \pm 0.22$ vs. $0.28 \pm$ 0.05 ; Fig. $1 \mathrm{C}, 1 \mathrm{D}$ and $2 \mathrm{~A}$ ). In a sub-analysis of mast cells in 6-week-old WAS and sham rats, according to colonic segment, a significantly higher number of mast cells in WAS rats was observed only in the proximal segment $(11.67 \pm 1.60$ vs. $5.96 \pm$ $0.71, P<0.05$; Fig. 2B). Chronic WAS also increased the number of mast cells in the distal segment; however, the difference did 


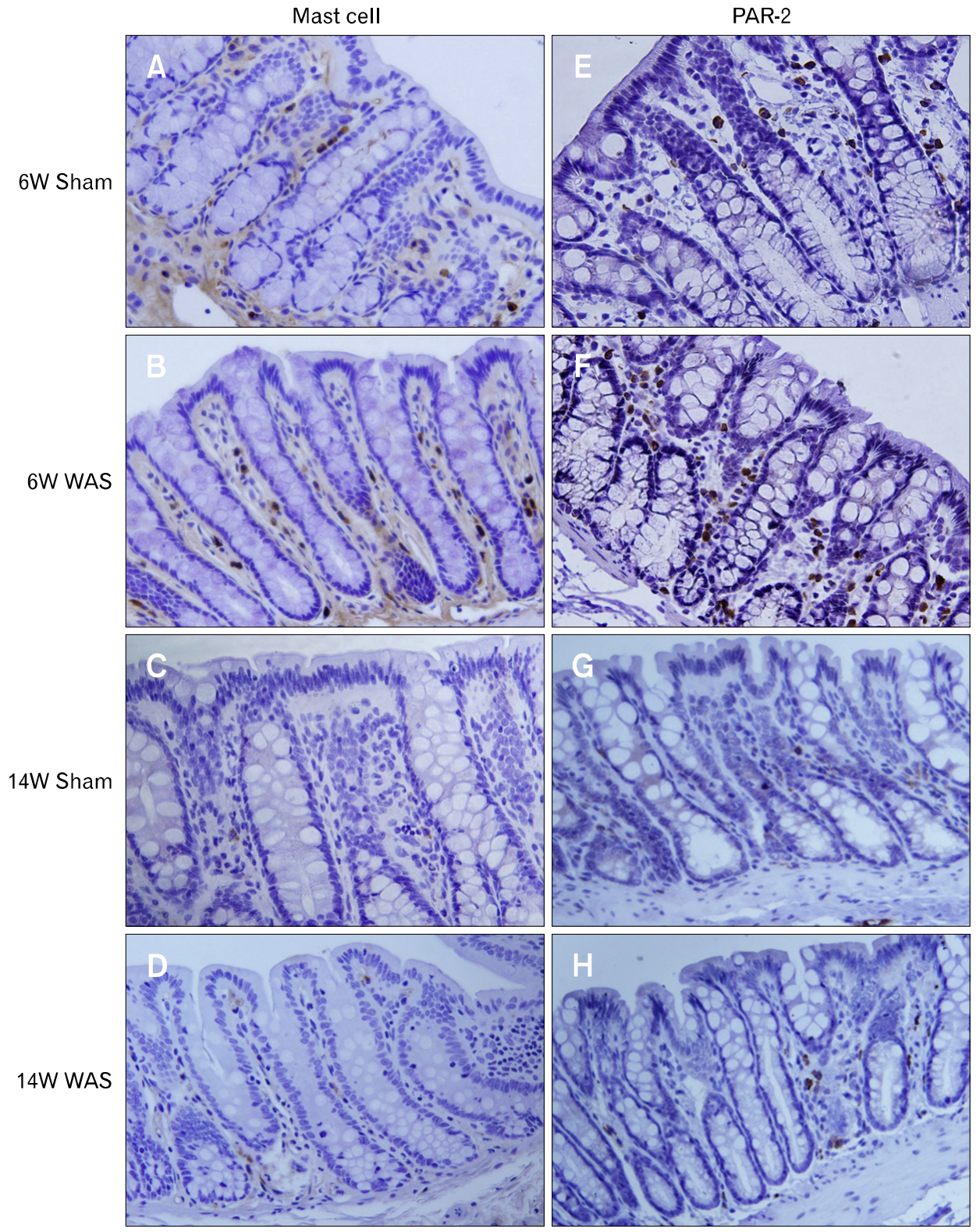

Figure 1. Immunohistochemical staining for tryptase (mast cells) and protease-activated receptor (PAR)-2 in colon sections of water avoidance stress (WAS)exposed and sham-exposed Ws/Ws rats. (A-D) WAS increased the number of mast cells in the lamina propria of the colonic mucosa of 6-week-old (6W) Ws/Ws rats, whereas mast cells were nearly absent in the colon of 14-weekold $(14 \mathrm{~W}) \mathrm{Ws} / \mathrm{Ws}$ rats. (E-H) The number of PAR-2-positive cells was significantly increased by WAS only in the 14-week-old rats (magnification, $\times 400$ ). Each photomicrograph is a representative of 6 rats/group. not reach statistical significance $(12.28 \pm 1.13$ vs. $9.30 \pm 1.30, P$ $=0.114$; Fig. 2C). In 14-week-old Ws/Ws rats, mast cells were nearly absent in the colonic mucosa and there were no statistical differences between WAS and sham rats in all segments.

\section{Changes in Protease-activated Receptor-2- positive Cell Numbers in the Colon}

In contrast to what was observed with mast cells, the number of PAR-2-positive cells in the whole colon ( $10.30 \pm 1.00$ vs. 8.88 $\pm 1.36, P>0.05$; Fig. $1 \mathrm{E}, 1 \mathrm{~F}$ and $3 \mathrm{~A}$ ) or in each colonic segment in 6-week-old rats was not significantly different between the WAS and sham group (Fig. 3B and 3C). Interestingly, in 14-week-old rats, a significantly higher number of PAR-2-positive cells was observed in the colonic mucosa of rats in the WAS group than in the sham group $(4.95 \pm 0.57$ vs. $3.13 \pm 0.26, P<$ 0.05; Fig. $1 \mathrm{G}, 1 \mathrm{H}$ and $3 \mathrm{~A}$ ). In a sub-analysis of PAR-2-positive cells in 14-week-old WAS and sham rats according to colonic segment, significantly higher number of PAR-2-positive cells in WAS rats was only observed in the proximal colon $(5.79 \pm 0.63$ vs. $2.90 \pm 0.33, P<0.01$; Fig. 3B). Some mast cells express not only tryptase but also PAR-2. Therefore, the number of the PAR-2-positive mast cells among the total PAR-2-positive cells 

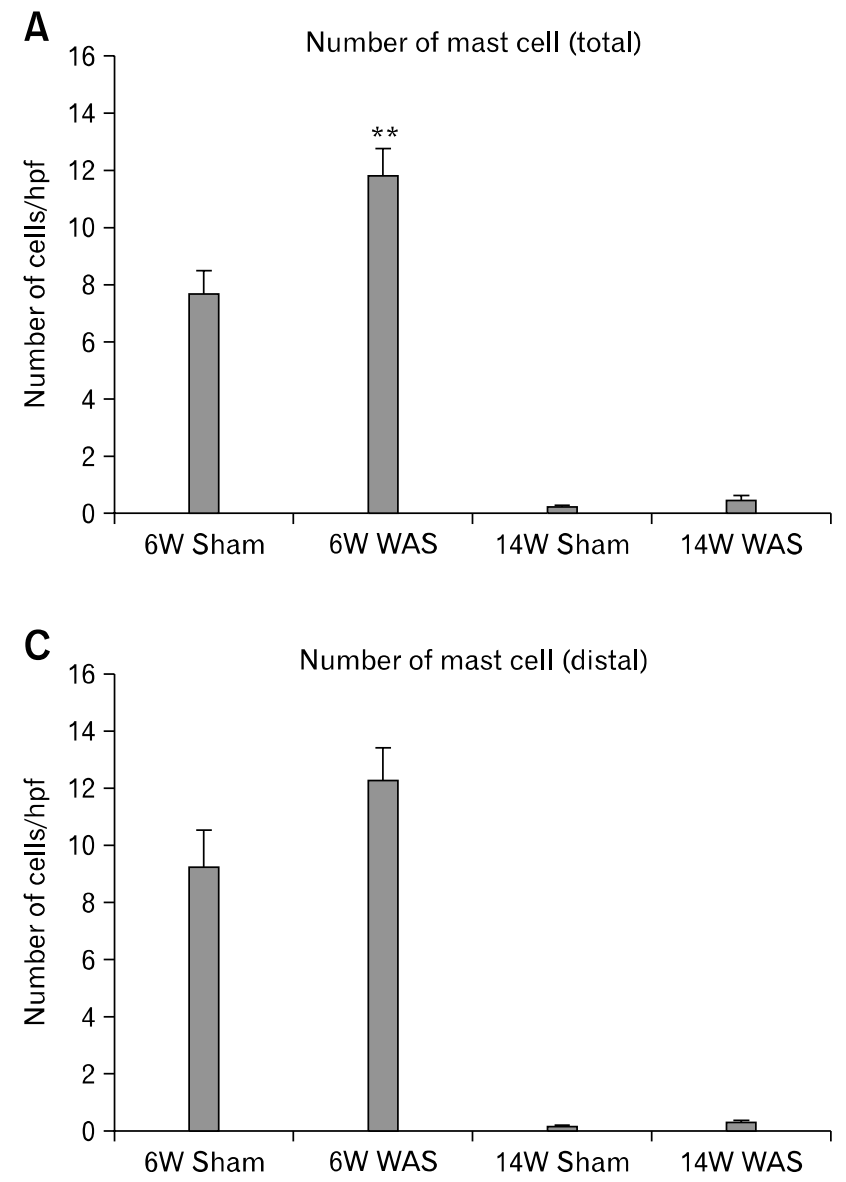

could be indirectly estimated by subtracting the number of PAR-2-positive cells in the 14-week-old group from that in the 6-week-old group. This indirect estimation indicated that WAS did not significantly increase the number of PAR-2-positive mast cells in the colonic mucosa of 6-week-old rats compared to the number in the sham group (Fig. 4).

\section{Change in the Fecal Pellet Output During Chronic Psychological Stress}

There was no difference in body weight changes between the WAS and sham groups during the experiment. Propulsive colonic motility, which was assessed by counting the number of fecal pellets expelled during the stress period, was higher following exposure to stress than following sham treatment (Fig. 5). This effect was significant for both 6-week-old and 14-week-old rats. The mean numbers of fecal pellets expelled daily by WAS and sham rats over the 13-days experimental period were $10.18 \pm$ 0.48 and $4.72 \pm 0.51$ pellets/hr in the 6-week-old rats, and 7.95 \pm 0.50 and $3.79 \pm 0.44$ pellets/hour in the 14 -week-old rats, respectively, (mean $\pm \mathrm{SEM}, P<0.05$ for both group). The rats

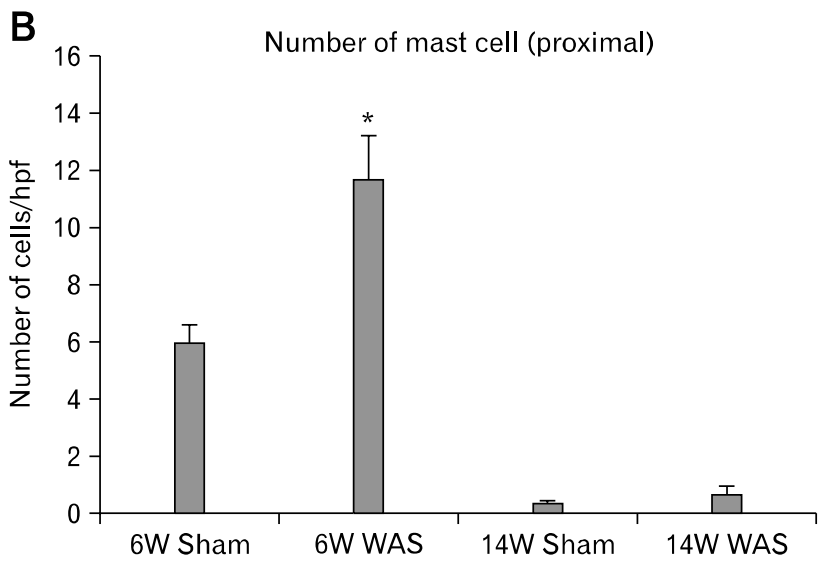

Figure 2. Changes in the number of mast cells in the colon of water avoidance stress (WAS)- and sham-treated Ws/Ws rats. (A) WAS increased the number of mast cells in the total colonic mucosa of 6-week-old (6W) rats. (B) In a sub-analysis according to colonic segment, the number of mast cells was significantly increased following WAS only in the proximal colon. (C) Although the number of mast cells also increased in the distal colon, it did not reach statistical significance. hpf, high power field; WAS, water avoidance stress; 14W, 14 -week-old. ${ }^{*} P<0.05$ vs. sham, ${ }^{* *} P<0.01$ vs. sham.

in the WAS group expelled a consistently higher number of fecal pellets over the 13-days WAS period; however, rats in the sham group expelled a decreasing number of fecal pellets over time (Fig. 5A and 5B). Cumulative FPO between the WAS and sham groups was also significantly different in later period of the experiment (Fig. 5C and 5D).

\section{Discussion}

In this study, we found that the numbers of mast cells and other PAR-2-positive cells in the proximal colon of $\mathrm{Ws} / \mathrm{Ws}$ rats were increased by chronic psychological stress. In addition, chronic psychological stress continuously increased colonic motor function regardless of the age of the rats or the presence of colonic mast cells.

Mast cells are known to increase visceral pain and permeability. Stress increased colonic macromolecular flux and ion secretion in wild-type rats, but not in $\mathrm{W}_{\mathrm{s}} / \mathrm{W}_{\mathrm{s}}$ rats ${ }^{18}$ furthermore $\mathrm{W}_{\mathrm{s}} / \mathrm{W}_{\mathrm{s}}$ rats did not exhibit TNBS-induced visceral hypersensitivity. ${ }^{19}$ Although chronic psychological stress increased the number of 

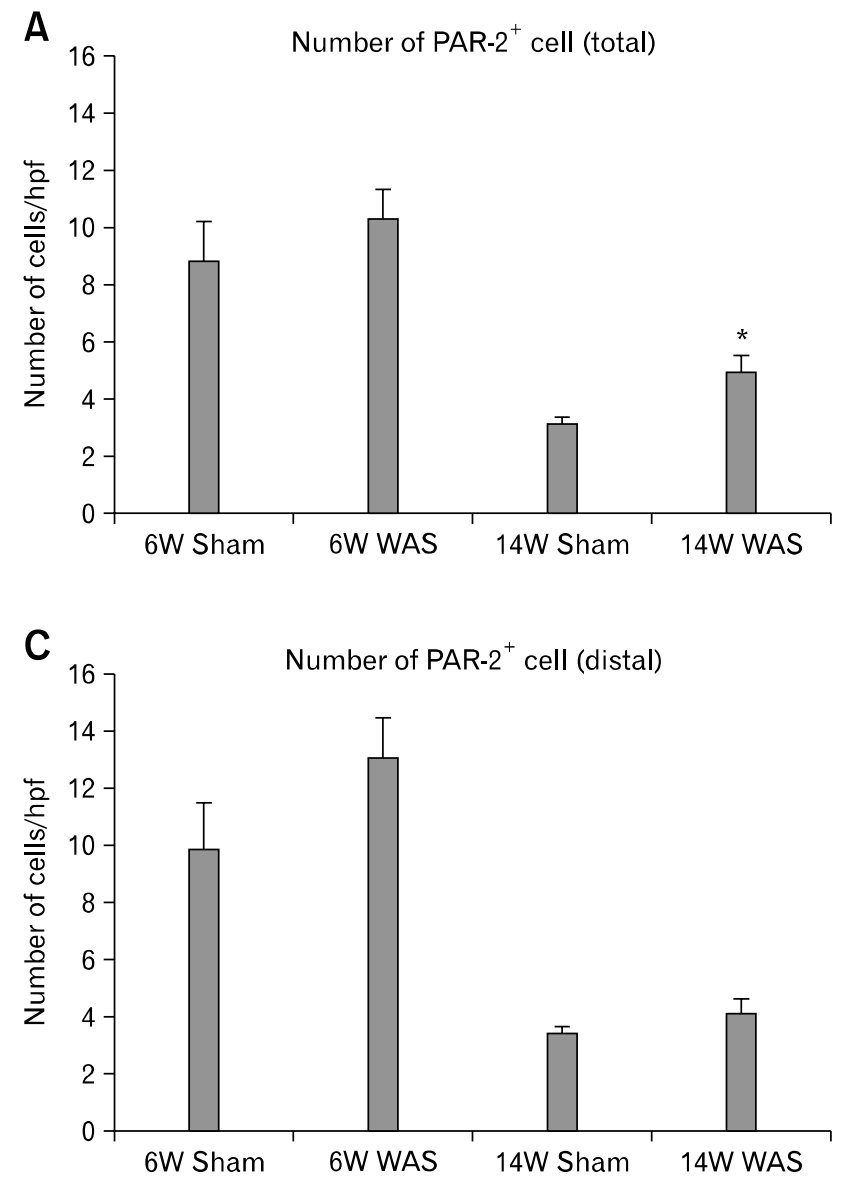

mast cells in the ileum and the colon of rats, ${ }^{18,20}$ the regional difference of this change within the colon has not been reported. In this study, we observed that the number of mast cells was increased by chronic WAS, and this increase was more prominent in the proximal colon than in the distal colon. The physiological functions of the proximal colon are distinct from those of the distal colon; the proximal colon acts as a reservoir and absorbs water, electrolytes, and nutrients. ${ }^{21}$ Mast cell activation leads to significant inhibition of water, $\mathrm{Na}^{+}, \mathrm{Cl}^{-}$and $\mathrm{K}^{+}$absorption in the proximal colon of antigen-sensitized rats. ${ }^{22}$ Therefore, the increased number of mast cells we observed in the proximal colon after repetitive stress, together with increased colonic motility, might play an important role in the development of loose stools or diarrhea in stress-related GI disorders.

However, our results from animal study are not always observed in the colonic mucosa of patient with IBS. Many human studies have shown increased mast cell in the colonic mucosa of patient with IBS, ${ }^{2,5-7}$ but the results were inconsistent and selective proximal mast cell proliferation was noted only in a few studies. ${ }^{2,7}$ This inconsistency is probably because the biopsy



Figure 3. Changes in the number of protease-activated receptor (PAR)-2-positive cells in the colon of water avoidance stress (WAS)and sham-treated Ws/Ws rats. (A-C) There was no difference in the number of PAR-2-positive cells in the colonic mucosa of 6-week-old $(6 \mathrm{~W})$ rats. (A, B) In 14-week-old (14W) rats, WAS increased the number of PAR-2-positive cells in the colonic mucosa, and, in a sub-analysis, this difference was only observed in the proximal colon. hpf, high power field. ${ }^{*} P<0.05$ vs. sham, ${ }^{* *} P<0.01$ vs. sham.

specimen was not taken from entire colon in most studies and the tissue sampling sites were different among studies such as only from the cecum, descending colon, or rectum. In addition, our findings indicated only the effect of psychological stress on mast cell proliferation in the colon, therefore proximal selectivity might not be found in patients with IBS who have complex pathogenesis, for example, visceral hypersensitivity, post-infectious, altered intestinal microbiota, stress and psychological dysfunction. ${ }^{23}$

The mechanism underlying the regional difference in mast cell proliferation caused by psychological stress is not clear. One plausible explanation is that the variation in proliferation of mast cells after stress might be related to differences in the innervation of the proximal and distal colon. Vagus nerves are the main source of parasympathetic innervation in the proximal colon, whereas sacral parasympathetic nerves innervate the distal colon. ${ }^{21}$ Vagus nerve has a role in the transmission of stress effect from central nervous system to GI tract. ${ }^{24}$ In addition, vagus nerve is recently proposed as one of the pathways involved in bi-directional communication between the gut microbiota and the brain, modulating brain-gut axis and GI immune function. ${ }^{25}$ 



Several studies indicate that vagal activation is related to activation and degranulation of mast cells in the stomach and ileum. Ketotifen and zinc sulfate, 2 mast cell stabilizers, inhibited the development of vagal-mediated gastric mucosal lesions, ${ }^{26,27}$ and electrical vagal stimulation increased histamine release in the guinea pig ileum. ${ }^{28}$ Therefore, vagal activation by repeated psychological stress might be a reason for the observed increase in mast cells in the proximal colon.

However, it was recently shown that the vagus nerve can modulate the systemic immune response and control inflammation in the settings of infection, injury, or sepsis. ${ }^{29}$ This mechanism is known as the "nicotinic anti-inflammatory pathway" because it is dependent on the $\alpha 7$-nicotinic acetylcholine receptor $(\alpha 7 \mathrm{nAChR})$ on macrophages. ${ }^{29}$ In our study, the type of stress was purely psychological, and the number of mast cells increased after repeated WAS. Based on these results, we can speculate that the vagus nerve may react differently to interoceptive stress (e.g., infection, trauma and shock) and exteroceptive stress (e.g., psychological stress). The exact role of the vagus nerve in the stress response should be elucidated in future studies.

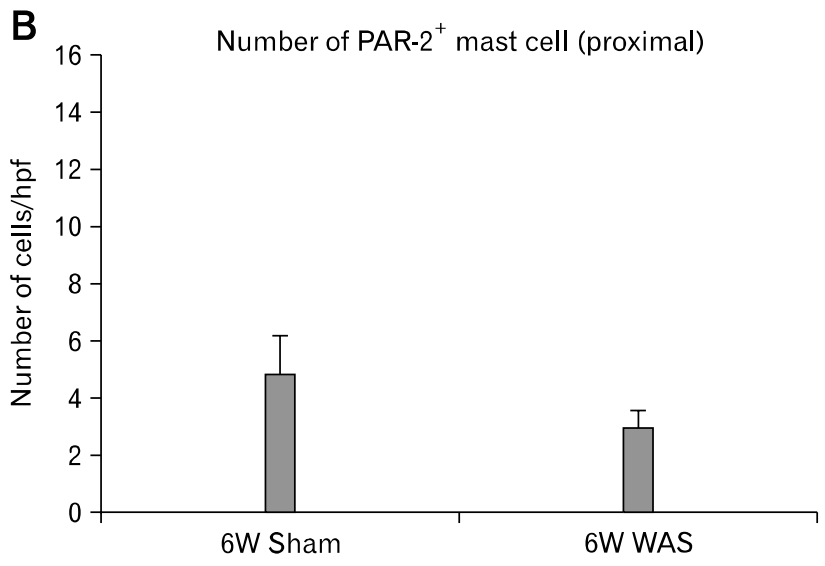

Figure 4. Estimated number of protease-activated receptor (PAR)-2-positive mast cells in the colon of water avoidance stress (WAS)- and sham-treated Ws/Ws rats. The number of PAR-2positive mast cells in the total, proximal, and distal colon was calculated by subtracting the number of PAR-2-positive cells in the 14-week-old group from that in the 6-week-old (6W) group. (A) There was no significant difference in the estimated number of PAR-2-positive mast cells between the WAS- and sham-treated groups. (B, C) In a sub-analysis according to colonic segment, there was no significant difference in the estimated number of PAR-2-positive mast cells between the proximal and distal colon. hpf, high power field.

PARs are seven trans-membrane-spanning, G-protein-coupled receptors and are highly expressed throughout the GI tract, which suggests their physiological and pathophysiological role in digestive diseases. ${ }^{30}$ The PAR-2 is known to be involved in intestinal permeability, visceral sensation, and smooth muscle contractility. ${ }^{9,10}$ The effects of mast cells on permeability and neuronal excitability are mediated by the PAR-2. ${ }^{31}$ Mast cells also express the PAR-2 and activate themselves through this receptor in an autocrine manner. ${ }^{32}$ Our results showed that chronic WAS increased the number of PAR-2-positive cells in the colon which were not mast cells. We previously reported that $60.6 \%$ of tryptase-positive cells in the colonic mucosa of patients with ulcerative colitis express PAR-2, and this finding indicated that a large portion of the PAR-2-positive cells in the laminar propria are mast cells. ${ }^{33}$ In the present study, the indirectly estimated number of PAR-2positive mast cells in the sham and WAS groups did not differ significantly. Considering the tryptase staining results, WAS seemed to increase mainly the PAR-2-negative mast cells in the colonic mucosa. However, the indirect estimation of PAR-2-positive mast cells was made on the presumption that the number of 
A

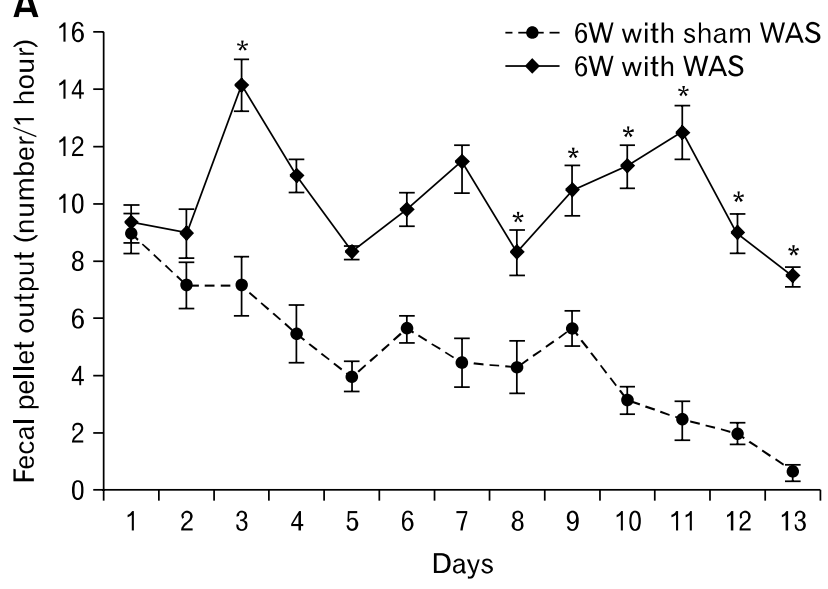

C

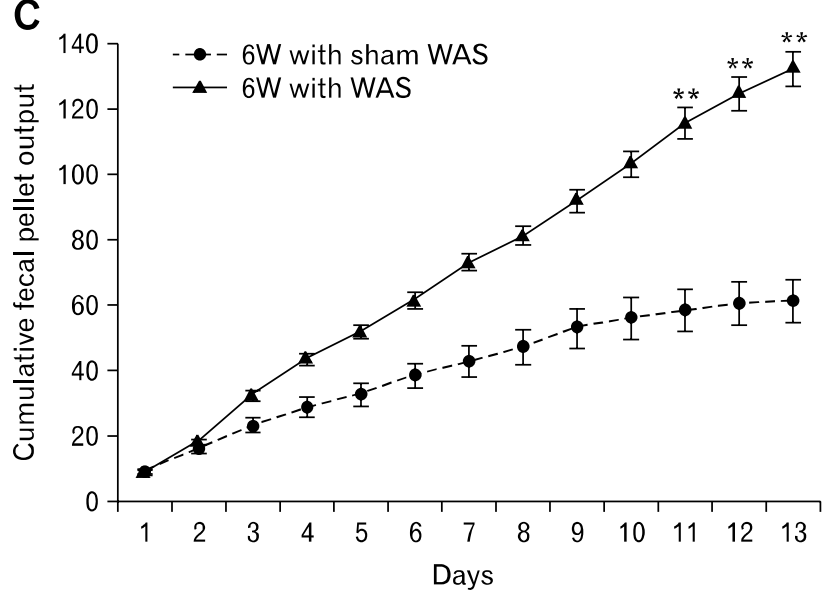

B

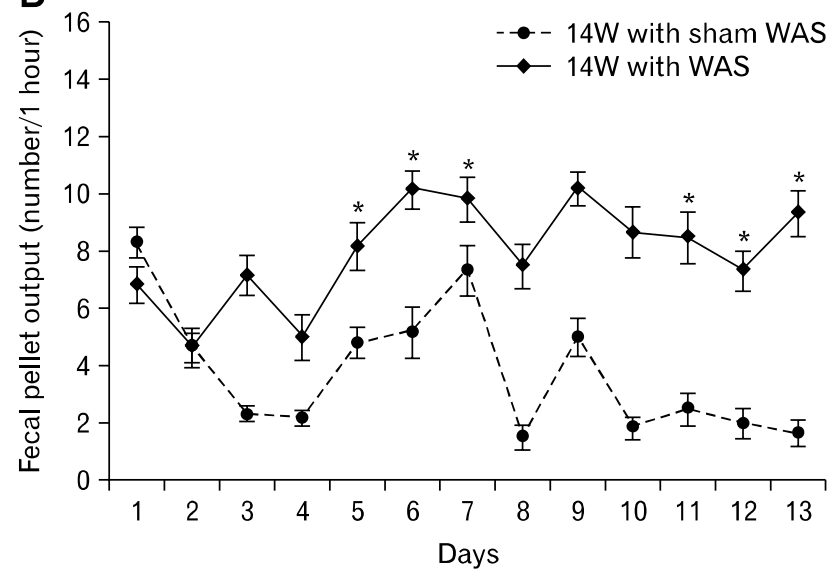

D

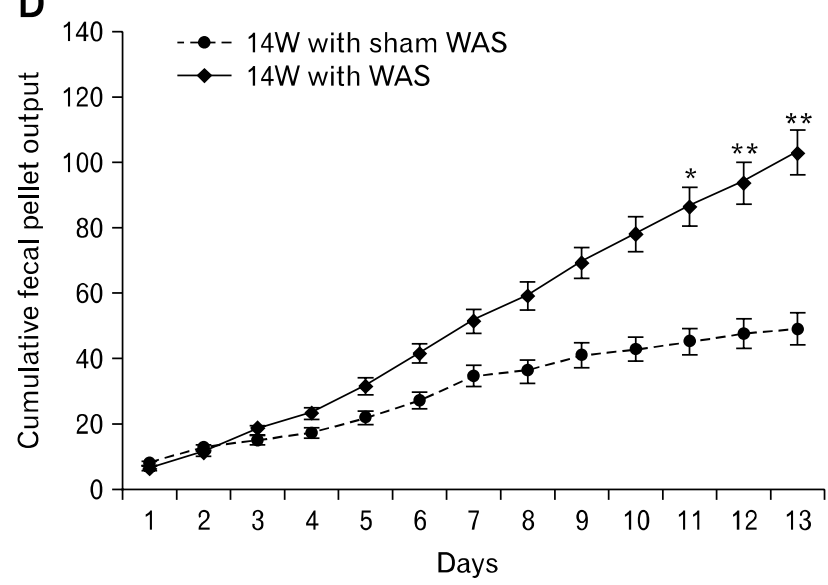

Figure 5. Change of fecal pellet output (FPO) during the water avoidance stress (WAS). (A, B) Daily FPO during the WAS experiment was increased in both 6-week-old (6W) and 14-week-old (14W) Ws/Ws rat. Rats exposed to repeated WAS exhibited a consistently high number of expelled pellets over the 13-days experimental period. In contrast, FPO decreased with time in the sham group. (C, D) Cumulative FPO during the WAS experiment differed significantly between 6 -week-old and 14 -week-old rats. ${ }^{*} P<0.05$ vs. sham, ${ }^{* *} P<0.01$ vs. sham.

PAR-2-positive non-mast cell of 6-week-old rat would increase in similar proportion to 14 -week-old rat. Therefore, it could have been miscalculated if the number of PAR-2-positive non-mast cells changed as the rats matured over time. In addition, the possibility of decrease in PAR-2-positive non-mast cell in WAS group of 6-week-old rat also could not be excluded. The change of PAR-2-positive mast cells should be measured directly using double staining for tryptase and PAR-2 in a future study.

In the current study, WAS significantly increased the number of PAR-2-positive cells in the 14-week-old Ws/Ws rats, which did not have mast cells. In addition to the mast cells, inflammatory cells, such as eosinophils and neutrophils, can express PAR-2, and they can be activated by the PAR-2 agonist trypsin. ${ }^{34,35}$ Therefore, our results indicate that chronic psychological stress increases not only mast cells but also other in- flammatory cells in the colon. Interestingly, similar to the change in mast cells following repeated WAS, PAR-2-positive cells were increased only in the proximal colon. Contrary to our results, acute restraint stress increased mast cells and PAR-2-positive cells in both the proximal and distal colon, suggesting that different mechanisms underlie the acute and chronic psychological stress responses. ${ }^{12}$

In the present study, the chronically stressed (WAS) group exhibited a continuously higher FPO compared to the non-stressed (sham) group in both the 6-week-old and 14-week-old Ws/Ws rats. Psychological stress stimulates colonic transit and defecation through the corticotropin-releasing factor 1 receptor. ${ }^{24}$ WAS activates the central stress pathway, including the paraventricular nucleus, the locus coeruleus, and Barrington's nucleus, and then the sacral parasympathetic nucleus, which innervates the de- 
scending colon, is stimulated. ${ }^{24}$ Previous reports showed that repeated WAS increases intestinal permeability and the number of mast cells in wild-type rats, but not in Ws/Ws rats which were older than 10-week-old. ${ }^{18,20}$ However, FPO from rats exposed to WAS increased in both wild-type and Ws/Ws rats compared with sham rats. ${ }^{18,20}$ Taken together with our results, this finding suggests that the colonic motor response to psychological stress is independent of mast cell activation and is more dependent on the neural pathway. Psychological stress like WAS increases CRF in the central nervous system and results in vagus nerve-mediated inhibition of gastric motility and sacral nerve-mediated stimulation of colonic motility. ${ }^{24}$ In contrast to motility changes, our study showed that WAS increased mast cell and PAR-2-positive cell number preferentially in the proximal colon. Because WAS activates both vagus and sacral parasympathetic nerve, this finding also implied the role of vagus nerve rather than sacral nerve in modulating GI immune function during stress response.

The rats in our study were not acclimated to the empty water tank, a new environment, before starting the WAS experiment, and this is why rats in both groups initially showed high FPO. FPO from the sham group decreased over time, whereas the WAS group continuously showed a high FPO during the entire experimental period. This finding implied that if subjects are chronically exposed to non-adaptable psychological stress, they might develop chronic colonic motor dysfunction leading to a functional GI disorder such as IBS.

The limitations of this study are that (1) we did not determine the exact type of PAR-2-positive cells in the colonic mucosa, and we did not directly measure how much this population overlapped with mast cells in 6-week-old Ws/Ws rats. The change of PAR-2positive mast cell should be elucidated using double staining for tryptase and PAR-2 in a future study; (2) we did not investigate the mechanism underlying the difference between the proximal and distal colon in the stress-mediated increase in mast cell and PAR-2positive cell numbers. Although the role of the vagus nerve in this regional difference was speculative, it is worthy to be elucidated in a future study; and (3) lastly, although we can avoid the effect of deficient of c-kit by comparing 6-week and 14-week-old Ws/Ws rats, different age between the groups could be another confounding factor. Therefore, appropriate control group should be selected according to the primary purpose of the experiment.

In summary, we showed that chronic psychological stress increased not only the number of mast cells but also other PAR-2-positive cells in the colonic mucosa of rats. This alteration was more prominent in the proximal colon than the distal colon, suggesting a regional difference in immune activation by chronic psychological stress. Therefore, the larger number of mast cells and PAR-2-positive non-mast cells in the proximal colon, together with parasympathetic mediated-increased colonic motility, might play an important role in the development of loose stools and/or diarrhea in patients with IBS exposed to chronic stress.

\section{References}

1. Ramsay DB, Stephen S, Borum M, Voltaggio L, Doman DB. Mast cells in gastrointestinal disease. Gastroenterol Hepatol (NY) 2010;6: 772-777.

2. O'Sullivan M, Clayton N, Breslin NP, et al. Increased mast cells in the irritable bowel syndrome. Neurogastroenterol Motil 2000;12:449-457.

3. Guilarte M, Santos J, de Torres I, et al. Diarrhoea-predominant IBS patients show mast cell activation and hyperplasia in the jejunum. Gut 2007;56:203-209.

4. Barbara G, Stanghellini V, De Giorgio R, et al. Activated mast cells in proximity to colonic nerves correlate with abdominal pain in irritable bowel syndrome. Gastroenterology 2004;126:693-702.

5. Cremon C, Gargano L, Morselli-Labate AM, et al. Mucosal immune activation in irritable bowel syndrome: gender-dependence and association with digestive symptoms. Am J Gastroenterol 2009;104:392-400.

6. Lee KJ, Kim YB, Kim JH, Kwon HC, Kim DK, Cho SW. The alteration of enterochromaffin cell, mast cell, and lamina propria $\mathrm{T}$ lymphocyte numbers in irritable bowel syndrome and its relationship with psychological factors. J Gastroenterol Hepatol 2008;23:1689-1694.

7. Piche T, Saint-Paul MC, Dainese R, et al. Mast cells and cellularity of the colonic mucosa correlated with fatigue and depression in irritable bowel syndrome. Gut 2008;57:468-473.

8. Molino M, Barnathan ES, Numerof R, et al. Interactions of mast cell tryptase with thrombin receptors and PAR-2. J Biol Chem 1997;272: 4043-4049.

9. Sato K, Ninomiya H, Ohkura S, Ozaki H, Nasu T. Impairment of PAR-2-mediated relaxation system in colonic smooth muscle after intestinal inflammation. Br J Pharmacol 2006;148:200-207.

10. Coelho AM, Vergnolle N, Guiard B, Fioramonti J, Bueno L. Proteinases and proteinase-activated receptor 2 : a possible role to promote visceral hyperalgesia in rats. Gastroenterology 2002;122:10351047.

11. Larauche M, Mulak A, Taché Y. Stress-related alterations of visceral sensation: animal models for irritable bowel syndrome study. J Neurogastroenterol Motil 2011;17:213-234.

12. Kim DH, Cho YJ, Kim JH, Kim YB, Lee KJ. Stress-induced alterations in mast cell numbers and proteinase-activated receptor-2 expression of the colon: role of corticotrophin-releasing factor. J Korean Med Sci 2010;25:1330-1335.

13. Tsujimura T, Hirota S, Nomura S, et al. Characterization of Ws mutant allele of rats: a 12-base deletion in tyrosine kinase domain of c-kit gene. Blood 1991;78:1942-1946.

14. Arizono N, Kasugai T, Yamada M, et al. Infection of Nippostrongylus brasiliensis induces development of mucosal-type but not connective tissue-type mast cells in genetically mast cell-deficient Ws/Ws rats. Blood 1993;81:2572-2578 
15. Niwa Y, Kasugai T, Ohno K, et al. Anemia and mast cell depletion in mutant rats that are homozygous at "white spotting (Ws)" locus. Blood 1991;78:1936-1941.

16. Takeda M, Takayama I, Terada N, et al. Immunoelectron-microscopic study of Kit-expressing cells in the jejunum of wildtype and Ws/Ws rats. Cell Tissue Res 2001;304:21-30.

17. Bradesi S, Schwetz I, Ennes HS, et al. Repeated exposure to water avoidance stress in rats: a new model for sustained visceral hyperalgesia. Am J Physiol Gastrointest Liver Physiol 2005;289:G42-G53.

18. Santos J, Yang PC, Söderholm JD, Benjamin M, Perdue MH. Role of mast cells in chronic stress induced colonic epithelial barrier dysfunction in the rat. Gut 2001;48:630-636.

19. Ohashi K, Sato Y, Kawai M, Kurebayashi Y. Abolishment of TNBS-induced visceral hypersensitivity in mast cell deficient rats. Life Sci 2008; 82:419-423.

20. Soderholm JD, Yang PC, Ceponis P, et al. Chronic stress induces mast cell-dependent bacterial adherence and initiates mucosal inflammation in rat intestine. Gastroenterology 2002;123:1099-1108.

21. Camilleri M, Ford MJ. Review article: colonic sensorimotor physiology in health, and its alteration in constipation and diarrhoeal disorders. Aliment Pharmacol Ther 1998;12:287-302.

22. Forbes D, Patrick M, Perdue M, Buret A, Gall DG. Intestinal anaphylaxis: in vivo and in vitro studies of the rat proximal colon. Am J Physiol 1988;255(2 Pt 1):G201-G205.

23. Tanaka Y, Kanazawa M, Fukudo S, Drossman DA. Biopsychosocial model of irritable bowel syndrome. J Neurogastroenterol Motil 2011; 17:131-139.

24. Taché $\mathrm{Y}$, Bonaz B. Corticotropin-releasing factor receptors and stressrelated alterations of gut motor function. J Clin Invest 2007;117: 33-40.

25. Cryan JF, Dinan TG. Mind-altering microorganisms: the impact of the gut microbiota on brain and behaviour. Nat Rev Neurosci 2012;13:701-712.

26. Cho $\mathrm{CH}$, Ogle $\mathrm{CW}$. The effects of zinc sulphate on vagal-induced mast cell changes and ulcers in the rat stomach. Eur J Pharmacol 1977;43:315-322

27. Király A, Süto G, Tam B, Hermann V, Mózsik G. Vagus-mediated activation of mucosal mast cells in the stomach: effect of ketotifen on gastric mucosal lesion formation and acid secretion induced by a high dose of intracisternal TRH analogue. J Physiol Paris 2000;94:131-134.

28. Bani-Sacchi T, Barattini M, Bianchi S, et al. The release of histamine by parasympathetic stimulation in guinea-pig auricle and rat ileum. J Physiol 1986;371:29-43.

29. Ulloa $\mathrm{L}$. The vagus nerve and the nicotinic anti-inflammatory pathway. Nat Rev Drug Discov 2005;4:673-684.

30. Vergnolle N. Review article: proteinase-activated receptors - novel signals for gastrointestinal pathophysiology. Aliment Pharmacol Ther 2000;14:257-266.

31. Jacob C, Yang PC, Darmoul D, et al. Mast cell tryptase controls paracellular permeability of the intestine. Role of protease-activated receptor 2 and beta-arrestins. J Biol Chem 2005;280:31936-31948.

32. He SH. Key role of mast cells and their major secretory products in inflammatory bowel disease. World J Gastroenterol 2004;10:309-318.

33. Kim JA, Choi SC, Yun KJ, et al. Expression of protease-activated receptor 2 in ulcerative colitis. Inflamm Bowel Dis 2003;9:224-229.

34. Miike S, McWilliam AS, Kita H. Trypsin induces activation and inflammatory mediator release from human eosinophils through protease-activated receptor-2. J Immunol 2001;167:6615-6622.

35. Howells GL, Macey MG, Chinni C, et al. Proteinase-activated receptor-2: expression by human neutrophils. J Cell Sci 1997;110 (Pt 7):881-887. 